\title{
Initial Hemodynamic Profiles of Children with Dengue Shock Syndrome in Referral Settings
}

\author{
Desy Rusmawatiningtyas ${ }^{1}$, Putu Aditya Wiguna ${ }^{3}$, Intan Fatah Kumara ${ }^{1}$, Nurnaningsih ${ }^{1}$, \\ Saptadi Yuliarto ${ }^{2}$, Eggi Arguni ${ }^{1}$, Antonius Pudjiadi ${ }^{4,}$, Sutaryo $^{1}$ \\ ${ }^{1}$ Department of Child Health, Faculty of Medicine, Public Health and Nursing, Universitas Gadjah Mada, Yogyakarta, Indonesia \\ ${ }^{2}$ Departement of Child Health, Faculty of Medicine, Universitas Brawijaya, DR. Saipul Anwar General Hospital, Malang, Indonesia \\ ${ }^{3}$ Academic Hospital-Universitas Mataram, Mataram, Indonesia \\ ${ }^{4}$ Departement of Child Health, Faculty of Medicine, Universitas Indonesia, Jakarta, Indonesia
}

\section{Email address:}

desy_rusma@yahoo.co.id (D. Rusmawatiningtyas), aditya.ku2004@gmail.com (P. A. Wiguna), intan.kumara@gmail.com (I. F. Kumara), nurnaningsih_pri@yahoo.com (Nurnaningsih), dr.saptadiyuliarto@gmail.com (S. Yuliarto), eggiarguni@ugm.ac.id (E. Arguni), ahpudjiadi@gmail.com (A. Pudjiadi), taryo@ugm.ac.id (Sutaryo)

${ }^{*}$ Corresponding author

\section{To cite this article:}

Desy Rusmawatiningtyas, Putu Aditya Wiguna, Intan Fatah Kumara, Nurnaningsih, Saptadi Yuliarto, Eggi Arguni, Antonius Pudjiadi, Sutaryo. Initial Hemodynamic Profiles of Children with Dengue Shock Syndrome in Referral Settings. American Journal of Pediatrics. Vol. 5, No. 4, 2019, pp. 260-266. doi: 10.11648/j.ajp.20190504.26

Received: October 16, 2019; Accepted: November 8, 2019; Published: November 19, 2019

\begin{abstract}
Background: Fluid therapy for dengue shock syndrome (DSS) requires a dynamic approach that involves monitoring of the pathophysiological processes as well as the preload, contractility, and afterload assessment during the course dengue infection. Hemodynamically unstable DSS patients received in referral setting often complicated by fluid overload and secondary infection. Objective: This study aims to provide hemodynamic profiles and fluid responsiveness of pediatric patients admitted to the PICU with DSS. Methods: Hemodynamic profiles, laboratories, and demographic data were collected from patients aged 1 month to 18 years old with DSS who were admitted to the Pediatric Intensive Care Unit (PICU) at Dr. Sardjito General Hospital, Yogyakarta, Indonesia from January to December 2016. Hemodynamic profiles were assessed in clinically shock and not clinically shock group at PICU admission using the non-invasive Ultrasonic Cardiac Output Monitor (USCOM). Fluid responsiveness in clinically shock group was evaluated after fluid challenge with $10 \mathrm{ml} / \mathrm{kgBW}$ crystalloid or colloid. Results: Eighty six subjects were included in this study. Sixty six subjects were admitted to PICU with clinically shock condition. This group received less intravenous fluid than hemodynamically stable group $(6.9 \mathrm{vs} 7.52 \mathrm{ml} / \mathrm{kgBW} / \mathrm{hour}$ respectively), had higher mean hematocrit level ( $42.09 \%$ vs $40.32 \%$ respectively), had higher hematocrit level during PICU stay $(43.37 \%$ vs $42.06 \%$ respectively), significantly higher percentage to receive inotropes agent $(62,1 \%$ vs $5 \%$, p 0,000$)$ and longer duration of inotropes usage (23,5 vs 0 hours, $\mathrm{p}$ 0.72). From the clinically shock patients admitted to PICU, only $19,69 \%$ were fluid responsive. Other subjects in this group with fluid non responsive state, 90,38\% had low inotropic index and high systemic vascular resistance index. Among 8 patients in clinically shock group who died during PICU stay, 6 of them had low cardiac Index, fluid non responsive condition, low inotropic index and high systemic vascular resistance index. Conclusion: Only a small percentage of DSS patients with clinically shock admitted to the PICU were fluid responsive. Majority of DSS cases in children had low inotropy index and high systemic vascular resistance index.
\end{abstract}

Keywords: Dengue Shock Syndrome, Initial Hemodynamic Profiles

\section{Introduction}

Dengue shock syndrome (DSS) has been the most common diagnosis of patient admitted to the pediatric intensive care unit (PICU) for the last 2 consecutive years (2015-2016). [1] The number of dengue cases reported annually to World Health Organization (WHO) had continued to increase from 0.4 million in 1996 to 1.3 million 
in 2005, 2.2 million in 2010, and 3.2 million in 2015. [2] In 2013, dengue was estimated to be responsible for approximately 3.2 million severe cases and 9000 deaths, the majority of which occurred in lower middle-income countries. [3, 4]

The WHO has issued a guideline for DSS management. Fluids should be administered as rapid (in less than 20 minute) intravenous (IV) bolus in a dose of $10-20 \mathrm{ml} / \mathrm{kg}$ body weight $(\mathrm{BW})$. When shock persists and the hematocrit is rising, a rapid bolus of plasma, plasma substitutes, or albumin should be repeated as necessary to a total colloid dose of 20-30 ml $/ \mathrm{kg}$. On the other hand, when shock persists but the hematocrit decreases, fresh whole-blood transfusions may be required $(10 \mathrm{ml} / \mathrm{kg})$. It is important to reduce the amount of IV fluids once the patient is recovering because over-hydration can result in intravascular fluid overload once the vascular permeability reverses along with the recovery. Shock can develop rapidly and, therefore, close monitoring is essential in DSS cases. [4]

Recent data suggests that early aggressive resuscitation on critically ill patients with shock may limit and/or reverse tissue hypoxia, prevent the progression to organ failures, and improve outcomes. However, excessive fluid has been associated with increased probability of complications, increased length of intensive care unit (ICU) and hospital stay, and increased mortality. [5] Unfortunately, there is still limited data about hemodynamic profile in DSS cases.

An USCOM, or Ultrasound Cardiac Output Monitoring, is a non-invasive device using Doppler ultrasonography method for assessing blood flow. It provides the clinicians the parameters of hemodynamic such as cardiac index (CI) and stroke volume (SV). [6, 7] In selective DSS cases in which thrombocytopenia is constantly present and the patients are unresponsive to fluid therapy, close monitoring of hemodynamic profiles is mandatory as it can help the physician to determine the next treatment with preferably non-invasive tools.

In referral settings, clinicians frequently received advanced cases of DSS where the patients have been complicated by fluid overload or other (dual) infections and have become hemodynamically unstable. To date, the evaluation of dengue cases is usually done clinically. This practice is prone to error then fails to provide clinicians with precise data about the patient's hemodynamic profile. [7] The aims of this study are to identify (1) the hemodynamic profiles of DSS patients in referral setting and (2) the percentage of patients in fluid responsive state in DSS patients using USCOM.

\section{Methods}

Data were collected using the consecutive sampling method from 97 children admitted to the PICU with DSS from 1 January 2016 to 31 July 2016. The study took place at Dr. Sardjito General Hospital, a tertiary-level university hospital in Yogyakarta, Indonesia. All subjects in this study were referral cases and have been previously treated in other lower-level health facilities in this region. The PICU in this hospital is a multidisciplinary 11-bed unit. Patients are taken care by 2 pediatric intensivists, 1 general pediatrician, and 10 pediatric residents, all of which have already completed the Pediatrics Advanced Life Support (PALS) course. Demographic data, hemodynamic profiles, and PICU outcomes of each patient were documented prospectively during the PICU stay.

\subsection{Study Population}

The inclusion criteria of this study were (1) patients aged 1 month to 18 year old who were admitted to the PICU, (2) matched the clinical criteria for dengue infection with shock syndrome, and (3) serologically confirmed for dengue infection.

Dengue infection was defined as acute fever with at least two of the following symptoms: headache, ocular pain, myalgia, arthralgia, rash, a positive tourniquet test (defined as the presence of 20 petechiae per 1 square inch), or leukopenia (defined as a white blood cell count of $<4.0 \times 10^{3}$ cells $/ \mu \mathrm{L})$. [2] A dengue infection was serologically confirmed by the presence of dengue $\operatorname{IgM}$ and $\operatorname{IgG}$ within a serum sample taken as early as the $5^{\text {th }}$ day of fever. The method used for this confirmation was enzyme-linked immunosorbent assays (ELISA).

Clinically shock patients were defined when they had (1) a rapid and weak pulse with a narrow pulse pressure (PP) of $<$ $20 \mathrm{mmHg}$ or (2) a systolic blood pressure $<5^{\text {th }}$ percentile according to age with signs of tissue hypoperfusion, i.e. $<1$ $\mathrm{mL} / \mathrm{kg} /$ hour of urine output (for patients whose body weights are less than $30 \mathrm{~kg}$ ) or $<0.5 \mathrm{~mL} / \mathrm{kg} /$ hour (for patients whose body weights are $30 \mathrm{~kg}$ and more), impaired consciousness, and cold or clammy skin. [18] Patients with underlying medical illnesses, including congenital cardiovascular problems, cerebrovascular diseases, epilepsy, lung diseases, liver diseases, kidney diseases, and autoimmune diseases were excluded from the study.

\subsection{Hemodynamic Assessment}

In this study, the hemodynamic parameters were measured using a Doppler ultrasound device (USCOM 1A, USCOM Pty Ltd, Coffs Harbor, NSW, Australia). This device projects a 2.2-MHz, continuous-wave, Doppler signal generated from a handheld transcutaneous probe placed at the projection of the aortic valve in a suprasternal position. The reflected signal is received back by the probe and analyzed by the base unit, which, with accurate signal processing, will later generate a real-time hemodynamic display. [7]

The USCOM utilizes the Doppler mode of traditional echocardiography to measure stroke volume (SV), ejection velocity, velocity time integral (VTI), and flow time (FT). This device can also calculate cardiac output (CO), cardiac index (CI), and systemic vascular resistance (SVR). SmithMadigan Inotropy Index (SMII) was calculated using a purpose-written computer program based on the formula developed by Brendan Smith and Veronica Madigan. [12] Further details can be seen on the manufacturer's website at 
www.uscom.com.au.

This study recorded all initial USCOM results in DSS patients immediately after admitted to the PICU and included patients with clinical signs of shock upon PICU arrivals. The patients with clinically shock condition upon PICU arrivals were allocated in the clinically shock group and therefore were given the fluid challenge test with $10 \mathrm{ml} / \mathrm{kgBW}$ crystalloid or colloid by their attending pediatric intensivists. The initial USCOM recording was performed simultaneously with the fluid challenge test. After completed the fluid challenge test, another USCOM examination was taken to check the patient's fluid responsiveness state. An increasing of more than $10 \%$ SVI from the initial result was presumed that the patient is responsive to the fluid challenge or in fluid responsiveness state. Unresponsive patients were then treated with appropriate vasoactive agents based on clinical signs and hemodynamic monitoring results. This study also recorded initial USCOM results of DSS patients admitted to PICU with no clinical signs of shock upon PICU arrivals.

\subsection{Outcome Measurement}

The USCOM measurement was performed by a pediatric intensivist or pediatric residents in charge. Another study had been done previously to evaluate inter-rater agreement of USCOM examination done by pediatric residents and pediatric intensivist, the result showed substantial agreement on SVI and moderate agreement on CI, SVRI and SMII parameters. Every USCOM measurement was taken with the aortic approach. This study used SMII to measure the inotropy component and SVRI to measure afterload component. For quality assurance, all USCOM examinations used in the analysis were verified by two of the authors. Admission demographic data, the time the shock diagnosis was made, the pediatric risk of mortality score (PRISM), and pediatric logistic organ dysfunction (PELOD) scores were recorded in addition to hemodynamic variables.

\subsection{Ethical Approval}

This study was approved by the medical and health research ethics committee (MHREC), Faculty of Medicine, Public Health, and Nursing, Universitas Gadjah Mada, Yogyakarta, Indonesia.

\section{Result}

A total of 271 children were admitted to PICU during the period of the study and $97(35.7 \%)$ among them had a primary diagnosis of DSS on PICU arrival. All children diagnosed with DSS were undergoing standard hemodynamic monitoring (electrocardiography and non-invasive blood pressure monitoring). From 97 observed DSS cases, 11 were excluded because the hemodynamic assessment with USCOM could not be performed soon after their PICU admissions. Patients who have clinical signs of shock upon PICU arrivals were classified into "group 1" and patients with no clinical signs of shock were classified into "group 2 ". The youngest patient was 5 months old while the oldest was 15 years and 4 months old. The distribution of the age, sex, length of PICU stay, PRISM III, PELOD, and clinical features upon PICU admission of patients from both groups are presented in Table 1 .

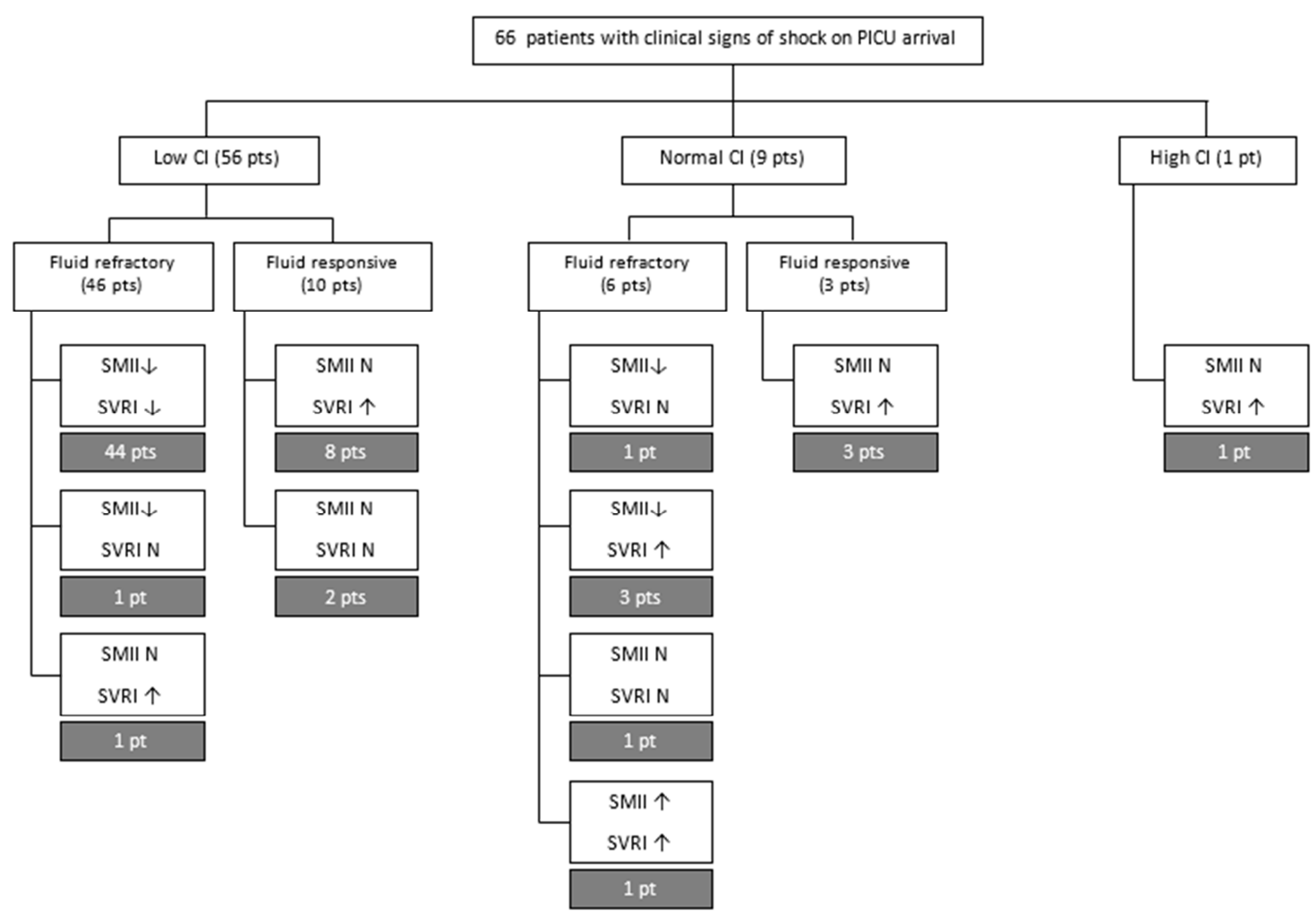

Figure 1. Hemodynamic profiles of 66 patients with clinical signs of shock upon PICU arrival. 
Table 1. Baseline characteristics.

\begin{tabular}{|c|c|c|c|}
\hline & Group $1(n=66)$ & Group $2(n=20)$ & $\mathbf{P}$ \\
\hline Mean age \pm DS (months) & $83.59 \pm 5.20$ & $90.00 \pm 9.77$ & 0.69 \\
\hline Male $(\%)$ & 57.60 & 35.00 & 0.07 \\
\hline Median (range) duration of fever when admitted (days) & $5(2-8)$ & $5(4-7)$ & 0.07 \\
\hline Median (range) time interval from the $1^{\text {st }}$ shock to PICU admission (hours) & $7.50(2.50-57.00)$ & $7.25(2-30)$ & 0.70 \\
\hline Mean ( \pm DS) hematocrit level upon PICU admission (\%) & $42.09 \pm 0.71$ & $40.32 \pm 1.06$ & 0.45 \\
\hline Mean ( \pm DS) highest hematocrit level during PICU stay $(\%)$ & $43.37 \pm 0.70$ & $42.06 \pm 1.13$ & 0.47 \\
\hline Median (range) PELOD score & $12(7-62)$ & $11(4-13)$ & 0.35 \\
\hline Inotrope use upon PICU admission (\%) & 62.10 & 15 & 0.00 \\
\hline Median (range) duration of inotrope use during PICU stay (hours) & $23.50(0-166)$ & $0(0-4)$ & 0.76 \\
\hline$\%$ of patients used more than 1 inotrope during PICU stay & 3 & 0 & 0.43 \\
\hline$\%$ of patients used mechanical ventilation during PICU stay & 27.30 & 5 & 0.03 \\
\hline$\%$ of patients received blood component transfusion during PICU stay & 53 & 35 & 0.15 \\
\hline Median (range) PICU LOS (days) & $3(1-18)$ & $3(2-5)$ & 0.51 \\
\hline Survival rate $(\%)$ & 87.90 & 95.00 & 0.36 \\
\hline
\end{tabular}

Slightly more than three-quarter (76.7\%) of DSS patients arrived in PICU with clinically shock condition (group 1) while the rest $(23.3 \%)$ showed no clinical sign of shock (group 2). This study found that in group 1, patients had received less intravenous fluid prior to their admissions to the PICU compared to group 2 patients $(6.90$ vs. 7.52 $\mathrm{ml} / \mathrm{kgBW} /$ hour). Three patients in group 1 had not received any fluids before they were admitted to this hospital because of difficulties in obtaining intravenous access. On the other hand, there was one patient in group $2(\mathrm{BW} 13 \mathrm{~kg})$ who had received $700 \mathrm{~mL}$ crystalloid within 1 hour $(53.80$ $\mathrm{ml} / \mathrm{kgBW} /$ hour) prior to the admission to the emergency room.

Group 1 patients were characterized by higher mean hematocrit level upon PICU arrival (42.09\% vs. $40.32 \%)$ and higher value of the highest hematocrit level reached during the PICU stay $(43.37 \%$ vs. $42.06 \%)$. While the median PRISM score of both groups were similar (6), the range of such score were larger in group 1 with a maximum score of 36 compared to 13 in group 2 . On the other hand, the median PELOD score upon the PICU admission was slightly higher in group 1 (12 vs. 11).

The proportion of DSS patients having received inotropes prior to the PICU admission was significantly higher in group 1 than group $2(62.10 \%$ vs $5 \% ; p$. 0.00$)$. The median duration of inotrope usage during the PICU stay was remarkably higher in group 1, but the difference was not statistically significant ( 23.50 vs. 0 hours; $p 0.72)$. Two ( $3 \%)$ patients received more than one inotrope in group 1 and none did so in group 2. Blood component transfusions were given to more patients in group 1 than in group 2 (53\% vs. $35 \%)$.

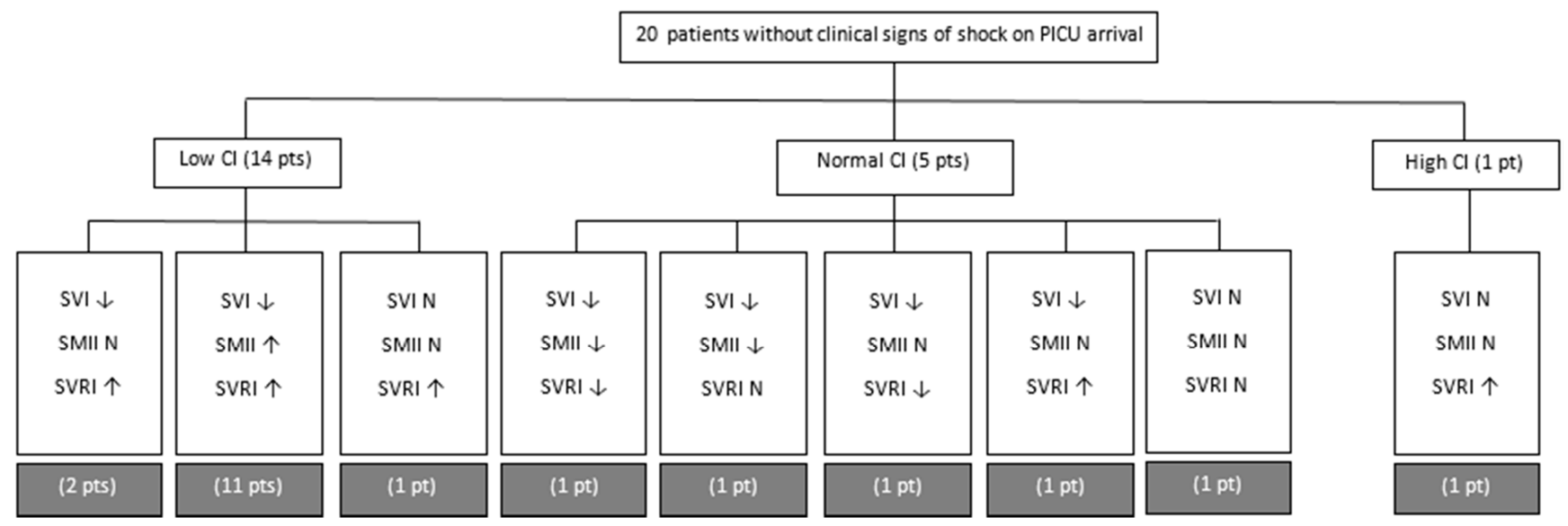

Figure 2. Hemodynamic profiles of 20 patients without clinical signs of shock upon PICU arrival.

The median length of PICU stay of both groups were 3 days. However, the longest length of stay of patients in group 1 could reach 18 days while the maximum length of stay of group 2 was 5 days. Patients in group 1 had lower survival rate $(87.90 \%$ vs. $95 \%)$.

In group 1 (Figure 2), the most common condition found was patient with low CI, fluid unresponsive state, low inotropy index and low SVRI in 46 patients from 66 patients.
Among 8 patients in group 1 who died during PICU stay, 6 had low CI, were fluid unresponsive, had low SMII nad had high SVRI.

Group 2 was comprised of 20 patients who showed no signs of shock upon PICU arrival and, therefore, did not receive the fluid challenge test. The most common condition found in group 2 was Low CI, fluid responsive state, increased inotropy index-increased SVRI. There were 3 
patients in group 2 who had already received inotrope infusions before the PICU admission. Six patients in this group were given another dose of fluid bolus during their PICU admissions. The only patient who died in group 2 had low CI-low SVI-low SMII-high SVRI.

\section{Discussion}

This was a prospective observational study among hospitalized children with DSS using USCOM, a noninvasive $\mathrm{CO}$ monitor based on 2D bedside ultrasonography. The mean age of patients in this study is within the age range when a child is most likely to be infected with dengue. This study is similar to a study in Vietnam in which DSS is more prevalent in male than female children. [13] This study also found that patients were commonly brought to the hospital on the fifth day of fever. The fourth to sixth day of fever are considered as the critical period of dengue because this is the phase when patients are highly prone to life-threatening complications, such as shock, bleeding, or organ failures.

As a tertiary referral institution, this hospital received advanced cases of DSS who need close hemodynamic monitoring. Proper monitoring can alert health care teams to an impending cardiovascular crisis before it develops into organ injuries. [14] Clinical examination alone is not sensitive enough to differentiate inotropic disorders from vascular disorders. Both disorders can be distinguished by measuring three basic hemodynamic components, i.e.: preload, contractility, and afterload, those are necessary to determine the $\mathrm{SV}, \mathrm{CO}$, and oxygen delivery $\left(\mathrm{DO}_{2}\right)$. Furthermore, knowing these three components will help guide fluid administration and determine the appropriate type and combination of vasoactive drugs for patients.

Pulmonary artery catheter thermodilution is to date the gold standard for $\mathrm{CO}$ measurement. Unfortunately, this method is not feasible in dengue patients with high bleeding risk due to its invasive nature. [15] Non-invasive hemodynamic monitoring would be more favorable for serial assessments of DSS patients. The use of non-invasive tool may facilitate better diagnosis, improve the efficacy of treatments, and give close monitoring for patients' response to the therapy given. However, the appropriate choice of a hemodynamic monitoring modality depends on the availability of facilities in the treating institution, the accuracy of the device, and the patient's conditions or contraindications. [16]

Recently, USCOM has been widely accepted and used as a bedside method to identify the type of shock and to help guide the fluid therapy for patients with shock. [17] However, its use in dengue cases has not been extensively investigated in Indonesia. Data obtained with the USCOM correlates well with data from other standard hemodynamic methods for animal studies, adults, neonates, and children. [8-11] The estimation of CO and SVRI measured with USCOMs are comparable to the results of pulmonary artery catheter thermodilution in children with normal cardiac anatomy. [11]
Therefore, this study used a non-invasive bedside hemodynamic monitor, to identify the initial hemodynamic profile and determine the fluid responsiveness of children with DSS.

The purpose of giving a patient a fluid challenge is to increase the SV (volume responsiveness). If the SV does not increase, the volume loading will no longer beneficial and may instead be harmful. Infusing fluid is the most direct way to challenge fluid responsiveness. [19] In a study where a "mini-fluid challenge" with $100 \mathrm{~mL}$ of colloid was performed to an adult population, the preload responsiveness is predicted by the changes in the velocity-time integral of the left ventricular outflow tract measured with echocardiography. [20] The statistical threshold was a $6 \%$ increase in the velocity-time integral. Nevertheless, since this threshold was below the precision of echocardiography, a $10 \%$ threshold was recommended even though it reduced the test accuracy. The main issue with the mini-fluid challenge is that small volumes of fluid can only induce small changes in the cardiac preload and, in patients with preload responsiveness, only small changes in the cardiac output. Thus, the test requires a very precise cardiac output monitoring system. Recently, some researchers have sought to determine the smallest volume of fluid required to perform an effective fluid challenge. In their study, a bolus of 4 $\mathrm{mL} / \mathrm{kg}$ over 5 minutes was the smallest volume that could reliably increase the mean circulating filling pressure and make fluid challenge interpretable in every circumstance. [21] This study used a $10 \%$ SVI increase from the USCOM measurement as the limit to assess fluid responsiveness. A fluid challenge test with $10 \mathrm{ml} / \mathrm{kgBW}$ crystalloid or colloid was used.

The study found that only one-fifth of patients with shock showed fluid responsiveness (figure 1). This might happen because the majority of patients had already received fluid resuscitation when they arrived at the PICU. According to the Frank-Starling principle, as the preload increases, the left ventricular (LV) SV increases until the optimal preload is achieved at which point the SV remains relatively constant. [22] This optimal preload is related to the maximal overlap of the actin-myosin myofibrils. Once the left ventricle is functioning near the "flat" part of the Frank-Starling curve, fluid loading has little effect on the stroke volume. In normal physiologic conditions, both ventricles operate on the ascending portion of the Frank-Starling curve. This mechanism provides a functional reserve to the heart in acute stress. In normal individuals, an increase in preload (with volume challenge) results in a significant increase in stroke volume. [5]

This study used SMII and SVRI to quantify cardiac inotropy and assess afterload. Smith and Madigan developed a hemodynamic theory-based formula to calculate the inotropy index, transferred from ventricle to the aorta, in patients undergoing a surgery or in critical conditions. Using a computer program, they later tested the formula against pre-stored data from 250 healthy subjects (as the control group) and 83 patients known to have acute left ventricular 
failure (the LVF group). They concluded that SMII may be used as a bedside tool to assess the inotropy status of patients in anesthesia and critical care. [12]

The study showed that the majority of DSS cases in children had low inotropy and high afterload. Inotropy degrees were measured using SMII parameters, while the afterload degree was measured using the SVRI). Low inotropy is usually followed by high afterload. Increased afterload occurs in response to a decrease in CI caused by catecholamine-related vasoconstriction or an increase in the systemic vascular resistance. It happens to maintain blood pressure and the distribution of blood and oxygen to vital organs such as the brain, heart, lung, and kidney. This compensatory mechanism is common in children and prevent hypotension until the advanced stage of shock. [23, 24]

In children, primary myocardial disorders can be found in cases of congenital or acquired heart defects. Meanwhile, secondary myocardial disorders could be caused by metabolic disorders, sepsis, and dengue shock syndrome. [25-28] Myocardial depression is also reported to occur in the toxic phase of grade I-II dengue and DSS in $13.8 \%$ and $36 \%$ of patients, respectively. The myocardial depression in these cases were characterized by ejection fractions (EF) of $<50 \%$. In addition, the CI in DSS usually ranges from 1.3 to $3.0 \mathrm{~L} / \mathrm{min} / \mathrm{m}^{2}$. [25] The mechanism of myocardial depression in dengue infection is still uncertain, but it has been known to result from hypoperfusion, the release of pro-inflammatory cytokines (TNF and IL-1), myocarditis, hypocalcemia, acidosis, or excess fluid. [25, 26]

This study also showed that most cases were in the fluid refractory state. The combination of fluid refractory state, low inotropy, and high afterload indicates a myocardial depression can increase the possibility of depression during advanced stages of shock. Fluid restriction and the use of inotropic drugs are logical approaches for patients with the aforementioned characteristics of shock. [14, 29]

There are several limitations of this study. The measurement of hemodynamic parameters was performed using the USCOM and the results were not validated by the gold standard. However, previous studies showed that parameters those obtained from USCOM correlate well with the accepted standard hemodynamic methods in children with normal cardiac anatomy. [30]

Since this study was done in a tertiary hospital, all subjects were referred from other health facilities and had been managed previously. Prior treatment may influence the patient's prognosis, and the severity of shock when the patients arrived in PICU. This study presents the initial hemodynamic profile of DSS in tertiary setting. The data from this study can be use as a basic for further treatment consideration. The various stage of shock shown by the subjects could represent the clinical situations happening in real practice.

\section{Conclusion}

Only a small percentage of DSS patients with clinically shock admitted to the PICU were fluid responsive. Majority of DSS cases in children had low inotropy index and high systemic vascular resistance index. Therefore, fluid therapy for DSS requires a dynamic approach that involves monitoring of the pathophysiological processes as well as the preload, contractility and afterload assessment during the critical course of a dengue infection. Providing physicians such clinical information will help improve the efficacy of their treatments and decrease the likelihood of complications due to dengue shock.

\section{Declaration of Interest}

All authors have no competing interest to declare. No author has any financial association with or received any funding from USCOM Ltd. or other organizations or individuals.

\section{References}

[1] Patient register annual data report Dr. Sardjito General Hospital 2017. Yogyakarta, Indonesia. 2017.

[2] World Health Organization. Global Strategy for Dengue Prevention and Control 2012-2020. World Health Organ. 2012; 43.

[3] Stanaway JD, Shepard DS, Undurraga EA, Halasa YA, Coffeng LE, Brady OJ, et al. The global burden of dengue: an analysis from the Global Burden of Disease Study 2013. Lancet Infect Dis. 2016; 16: 712-23.

[4] World Health Organization. Dengue: guidelines for diagnosis, treatment, prevention, and control. Spec Program Res Train Trop Dis. 2009; 43

[5] Alobaidi R, Morgan C, Basu RK, Stenson E, Feartherstone R, Majumdar SR, et al. Association between fluid balance and outcome in Critically ill children: A systematic review and meta-analysis. JAMA Pediatr. 2018.

[6] Chand R, Mehta Y, Trehan N. Cardiac output estimation with a new doppler device after off-pump coronary artery bypass surgery. J Cardiothorac Vasc Anesth. 2006; 20 (3): 315-9.

[7] Phillips R, Paradisis M, Evans N, Southwell D, Burstow D, West M. Cardiac output measurement in preterm neonates: validation of USCOM against echocardiography [abstract]. Crit Care. 2006; 10 (1 suppl): p343

[8] Chew MS, Poelaert J. Accuracy and repeatability of pediatric cardiac output measurement using Doppler: 20-year review of the literature. Intensive Care Med. 2003; 29: 1889-94.

[9] Dhanani S, Barrowman NJ, Ward RE, Murto KT. Intra- and inter-observer reliability using a noninvasive ultrasound cardiac output monitor in healthy anesthetized children. Pediatr Anesth. 2011; 21: 858-64.

[10] Cattermole GN, Leung PYM, Mak PSK, Chan SSW, Graham $\mathrm{CA}$, Rainer TH. The normal ranges of cardiovascular parameters in children measured using the Ultrasonic Cardiac Output Monitor. Crit Care Med. 2010; 38: 1875-81.

[11] Kuster M, Exadaktylos A, Schnuriger B. Non-Invasive hemodynamic monitoring in trauma patients. World J Emerg Surg. 2015; 10: 11 . 
[12] Madigan VM, Smith BE. Non-invasive method for rapid bedside estimation of inotropy: Theory and preliminary clinical validation. Br J Anaesth. 2013; 111: 580-8.

[13] Dinh The T, Le Thi Thu T, Nguyen Minh D, Tran Van N, Tran Tinh H, Nguyen Van Vinh C, et al. Clinical Features of Dengue in a Large Vietnamese Cohort: Intrinsically Lower Platelet Counts and Greater Risk for Bleeding in Adults than Children. Halstead SB, editor. PLoS Negl Trop Dis. 2012; 6: e1679.

[14] Rajapakse S, Rodrigo C, Rajapakse A. Treatment of dengue fever. Infect Drug Resist. 2012; 5: 103-12.

[15] Thanachartwet V, Wattanathum A, Sahassananda D, Wacharasint P, Chamnanchanunt S, Khine Kyaw E, et al. Dynamic Measurement of Hemodynamic Parameters and Cardiac Preload in Adults with Dengue: A Prospective Observational Study. Huy NT, editor. PLoS One. 2016; 11: e0156135.

[16] Moulton SL, Mulligan J, Srikiatkhachorn A, Kalayanarooj S, Grudic GZ, Green S, et al. State-of-the-art monitoring in treatment of dengue shock syndrome: a case series. J Med Case Rep. 2016; 10: 233.

[17] Li C, Lin F, Fu S, Chen G, Yang X, Zhu C, and others. Stroke volume variation for prediction of fluid responsiveness in patients undergoing gastrointestinal surgery. Int $\mathrm{J}$ Med Sci. 2013; 10 (2): 148-55.

[18] Critchley L, Peng Z, Fok B. Testing the reliability of a new ultrasonic cardiac output monitor, the USCOM, by using aortic flow probes in anesthetized dogs. Anesth Analg. 2005; 100 (3): 748-53.

[19] Vincent JL, Weil MH. Fluid challenge revisited. Crit Care Med. 2006; 34: 1333-7.

[20] Muller L, Toumi M, Bousquet PJ, Riu-Poulenc B, Louart G, Candela D, et al. An increase in aortic blood flow after an infusion of $100 \mathrm{ml}$ colloid over 1 minute can predict fluid responsiveness: the mini-fluid challenge study. Anesthesiology. 2011; 115: 541-7.
[21] Monnet X, Marik PE, Teboul JL. Prediction of fluid responsiveness: an update. Annals of intensive care. 2016; 6: 111-118.

[22] Nixon J, Murray R, Leonard P, Al. E. Effect of large variations in preload on left ventricular performance characteristics in normal subjects. Circulation. 1982; 65: 698-703.

[23] Arikan AA, Citak A. Pediatric shock. Signa Vitae. 2008; 3 : 13-23.

[24] Convertino VA, Wirt MD, Glenn JF, Lein BC. The compensatory reserve for early and accurate prediction of hemodynamic compromise: A review of the underlying physiology. Shock. 2016; 45: 580-90.

[25] Khongphatthanayothin A, Lertsapcharoen P, Supachokchaiwattana P, La-orkhun V, Khumtonvong A, Boonlarptaveechoke $\mathrm{C}$, et al. Myocardial depression in dengue hemorrhagic fever: Prevalence and clinical description. Pediatr Crit Care Med. 2007; 8: 524-9.

[26] Kirawittaya T, Yoon IK, Wichit S, Green S, Ennis FA, Gibbons R V., et al. Evaluation of cardiac involvement in children with dengue by serial echocardiographic studies. PLoS Negl Trop Dis. 2015; 9: 1-17.

[27] Teparrukkul P, Hantrakun V, Day NPJ, West TE, Limmathurotsakul D. Management and outcomes of severe dengue patients presenting with sepsis in a tropical country. PLoS One. 2017; 12: 1-13.

[28] Virk HUH, Inayat F, Ur Rahman Z. Complete heart block in association with dengue hemorrhagic fever. Korean Circ J. 2016; 46: 866-9.

[29] Wongsa A. Fluid and hemodynamic management in severe dengue. Southeast Asian J Trop Med Public Health. 2015; 46: $123-7$.

[30] Beltramo F, Menteer J, Razavi A, Khemani RG, Szmuszkovicz J, Newth CJL, et al. Validation of an Ultrasound Cardiac Output Monitor as a Bedside Tool for Pediatric Patients. Pediatr Cardiol. 2016; 37: 177-83. 